\title{
OXIDATION DYNAMICS OF NANOPHASE ALUMINUM CLUSTERS: A MOLECULAR DYNAMICS STUDY
}

\author{
Shuji Ogata ${ }^{a}$, Timothy J. Campbell ${ }^{b}$, Kenji Tsuruta ${ }^{b}$, Aiichiro Nakano ${ }^{b}$, \\ Rajiv K. Kalia ${ }^{b}$, Priya Vashishta ${ }^{b}$, and Chun-K. Loong ${ }^{c}$ \\ aDepartment of Applied Sciences, Yamaguchi University, 2557 Tokiwadai, Ube 755, JAPAN \\ ogata@po.cc.yamaguchi-u.ac.jp
}

${ }^{b}$ Concurrent Computing Laboratory for Materials Simulations Department of Physics and Astronomy, Department of Computer Science Louisiana State University, Baton Rouge, LA 70803 http://www.cclms.Isu.edu

'Argonne National Laboratory, Argonne, IL 60439

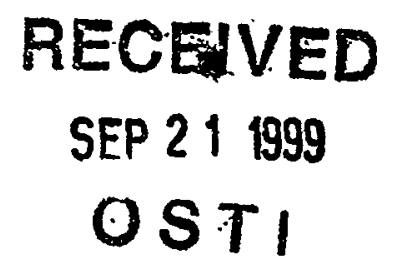

\section{ABSTRACT}

Oxidation of an aluminum nanocluster $(252,158$ atoms) of radius $100 \AA$ placed in gaseous oxygen $(530,727$ atoms) is investigated by performing molecular-dynamics simulations on parallel computers. The simulation takes into account the effect of charge transfer between $\mathrm{Al}$ and $\mathrm{O}$ based on the electronegativity equalization principles. We find that the oxidation starts at the surface of the cluster and the oxide layer grows to a thickness of $\sim 28 \AA$. Evolutions of local temperature and densities of $\mathrm{Al}$ and $\mathrm{O}$ are investigated. The surface oxide melts because of the high temperature resulting from the release of energy associated with Al-O bondings. Amorphous surface-oxides are obtained by quenching the cluster. Vibrational density-of-states for the surface oxide is analyzed through comparisons with those for crystalline $\mathrm{Al}, \mathrm{Al}$ nanocluster, and $\alpha-\mathrm{Al}_{2} \mathrm{O}_{3}$.

\section{INTRODUCTION}

Various kinds of solid materials including metals and certain semiconductors oxidize easily, resulting in alteration of their physical and mechanical properties [1]. The nanophase materials, produced by consolidating nanometer-sized clusters, have recently drawn much interest because of their novel properties. Aluminum nanoclusters of radii $70-200 \AA$ are known to form amorphous-alumina (i.e., $\mathrm{Al}_{\mathrm{x}} \mathrm{O}_{\mathrm{y}}$ ) layers limited to thickness of $20-30 \AA$ when they are exposed to an oxygen environment at room temperatures [2,3]. Alumina is one of the most widely used ceramics famous for its high hardness, high melting temperature, and high electric resistance. Passivated nanophase-Al [4-6], a consolidation of those passivated Al-nanoclusters, may retain such useful properties as well as high oxidation resistance.

In this paper we report on the first molecular-dynamics simulations for the oxidation of an Al-nanocluster (radius $100 \AA$ ). The simulation takes into account the process of dynamic charge transfer between $\mathrm{O}$ and $\mathrm{Al}$ on the basis of the electronegativity equalization principles $[7,8]$. We find that the oxidation starts from the surface of the cluster accompanied by substantial charge transfer between the atoms. The local temperature in the surface region rises owing to substantial release of Al-O bond-formation energies. In the initial stage of the oxidation process $(t=0-25 \mathrm{ps})$, the thickness of the surface oxide increases linearly with time. The surface temperature rises linearly with time to a value $2,300 \mathrm{~K}$ at $t=24 \mathrm{ps}$, which corresponds to the melting temperature of alumina. For $t>25 \mathrm{ps}$, the oxide layer saturates to $\sim 28 \AA$ at $t=30 \mathrm{ps}$. Continuation of the oxidation simulation results in thermal emission of fragmented oxides of various sizes. By quenching the melted surface-oxides at $t=35 \mathrm{ps}$, we obtain amorphous oxides covering the Al core. We calculate vibrational spectra of atoms in various radial shells of the 


\section{DISCLAIMER}

This report was prepared as an account of work sponsored by an agency of the United States Government. Neither the United States Government nor any agency thereof, nor any of their employees, make any warranty, express or implied, or assumes any legal liability or responsibility for the accuracy, completeness, or usefulness of any information, apparatus, product, or process disclosed, or represents that its use would not infringe privately owned rights. Reference herein to any specific commercial product, process, or service by trade name, trademark, manufacturer, or otherwise does not necessarily constitute or imply its endorsement, recommendation, or favoring by the United States Government or any agency thereof. The views and opinions of authors expressed herein do not necessarily state or reflect those of the United States Government or any agency thereof. 


\section{DISCLAIMER}

Portions of this document may be illegible in electronic image products. Images are produced from the best available original document. 
oxidized cluster. We thereby find that the spectra for the surface oxides have the characteristics of both crystalline $\mathrm{Al}$ and $\alpha-\mathrm{Al}_{2} \mathrm{O}_{3}$.

\section{PARALLEL MOLECULAR DYNAMIICS OF AI AND O SYSTEMS}

The principal difficulty in simulating large-scale $\mathrm{MD}$ of materials composed of $\mathrm{Al}$ and $\mathrm{O}$ stems from ionic nature of $\mathrm{Al}$ and $\mathrm{O}$. Substantial charge transfer takes place between these atoms due to their difference in electronegativity [9], which depends sensitively on the atomic configuration in the material [10]. Realization of MD simulations for such systems requires efficient ways of determining local atomic-charges $[7,8]$ and the resulting long-range Coulomb interactions.

In the present simulations, we adopt the empirical interatomic potentials developed by Streitz and Mintmire [7] for aluminum and alumina systems (referred to ES+). In the ES+ model, local atomic-charges vary with the environment in accordance with the electronegativity equalization principles. With those potentials, we can reproduce cohesive energies [7], elastic constants [7], and phonon spectra [11]. Furthermore the surface energies [7] for low-index faces of $\alpha-\mathrm{Al}_{2} \mathrm{O}_{3}$ predicted by the model agree well with those obtained by the first-principles calculations [10]. The Coulomb interaction is computed efficiently with the fast-multipole method (FMM) $[12,13]$ with a useful extension for the local stress calculations [14]. The.FMM enables us to calculate the Coulomb interactions in $\mathrm{O}(N)$ operations; multipoles upto the quadrupole are taken in the FMM.

Present MD code is highly parallelized; the parallel efficiency is close to unity. All the simulations are performed on 40-node DEC-Alpha 4/175 cluster interconnected by two Gigaswitches and on 8-node DEC-Alpha 5/500 cluster interconnected by Fast Ethernet in the Concurrent Computing Laboratory for Materials Simulations at Louisiana State University.
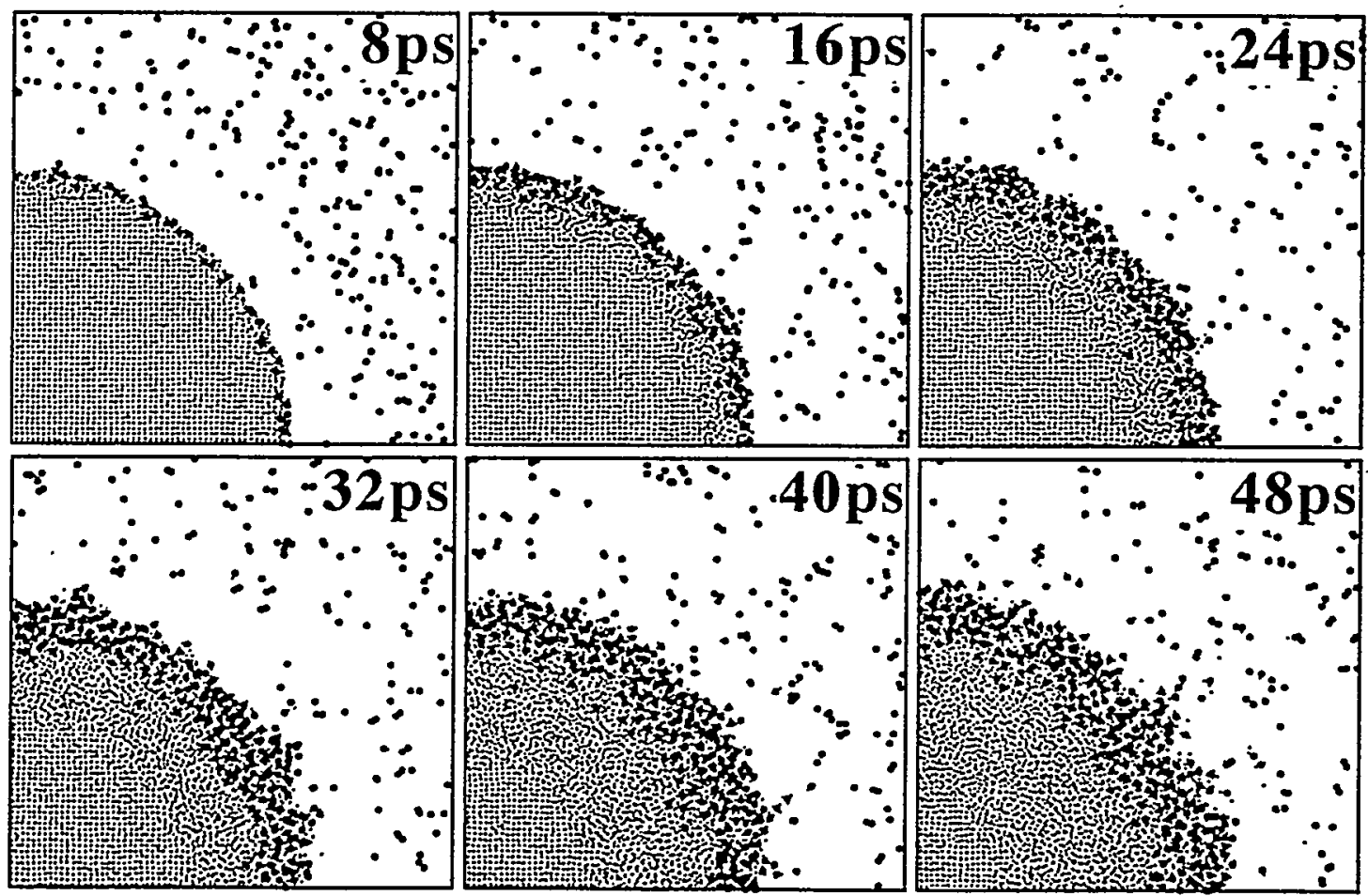

Fig 1: The $x-y$ projection maps of a slice $(x=0-160 \AA, y=0-160 \AA, z=0-8 \AA)$ at various times. The larger spheres correspond to $\mathrm{O}$; the smaller to Al. 


\section{OXIDATION DYNAMICS OF NANOPHASE ALUMINUM CLUSTERS}

A fcc-crystalline Al-nanosphere of radius $100 \AA$ composed of 252,158 atoms is placed at the center $(x=y=z=0 \AA)$ of the MD box $(800 \AA \times 800 \AA \times 800 \AA)$. The Al nanocluster is equilibrated at $300 \mathrm{~K}$. The cluster is surrounded by 530,727 oxygen atoms distributed randomly in an annulus with radii $r=110-400 \AA$ from the center of the MD box. A spherical hard wall of radius $400 \AA$ is used to confine the system. Density of $\mathrm{O}$ is about 41 times that of $\mathrm{O}_{2}$ gas in the standard state (i.e., $1 \mathrm{~atm}$ and $300 \mathrm{~K}$ ). Starting with the initial velocities of $\mathrm{O}$ atoms corresponding to $300 \mathrm{~K}$, we integrate the Newton's equation of motion using the velocity-Verlet algorithm [15]. To accelerate computations, we adopt multiple-time steps [15] of lfs for short-range forces $(<6 \AA)$ and 20 fs for long-range forces.

Figures 1 display $x-y$ projection maps of a slice $(x=0-160 \AA, y=0-160 \AA, z=0-8 \AA)$ at $t=8$, $16,24,32,40$, and 48ps; the left bottom corner in each map corresponds to the center $(x=y=z=0 \AA)$. Larger spheres in Fig. 1 correspond to $O$, smaller spheres to Al. Figure 2 depicts (top) spherically averaged temperatures, (middle) radial densities of $\mathrm{O}$, and (bottom) radial densities of Al in every $10 \AA$-shell at $t=8,24$, and 40 ps.

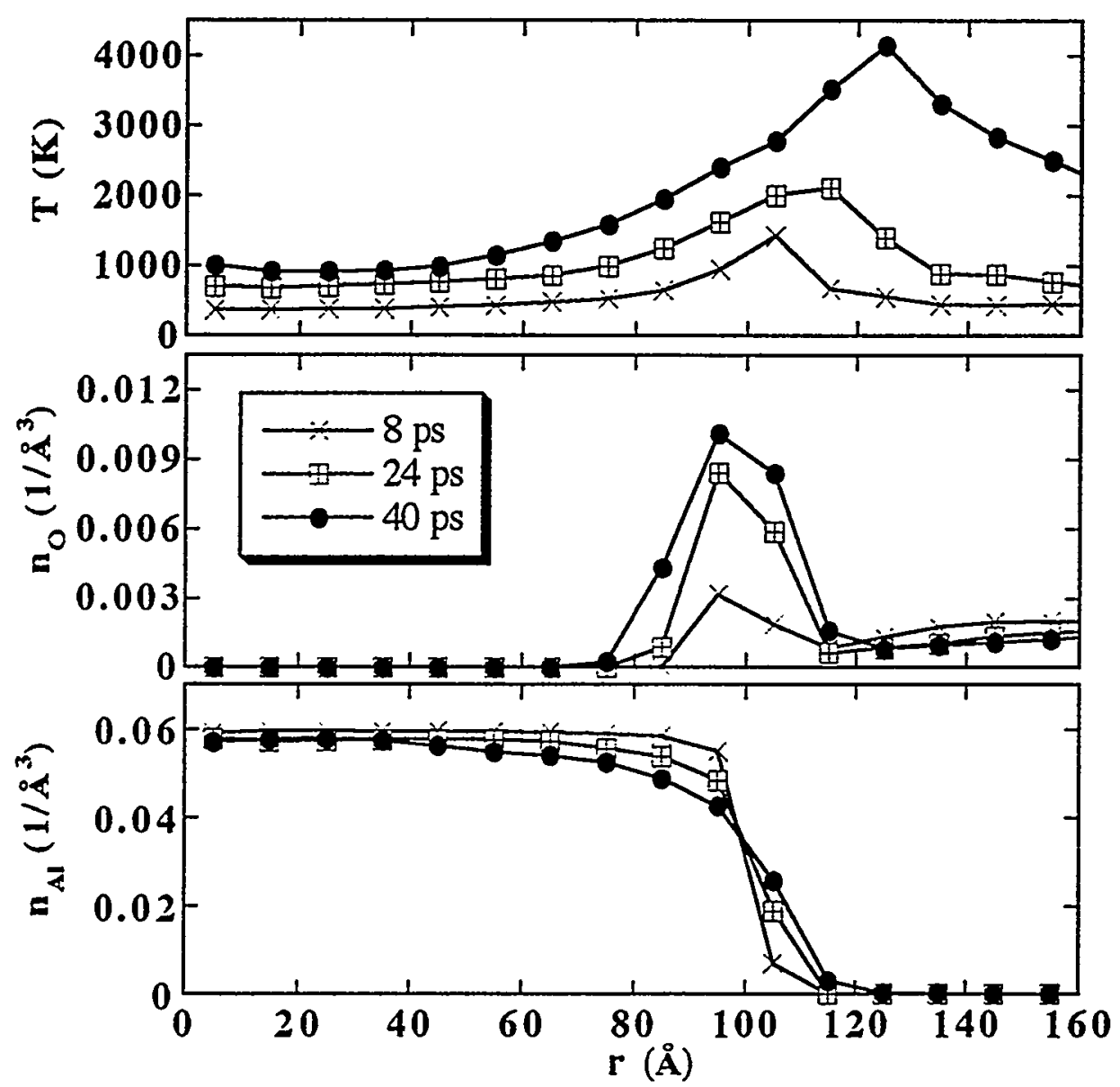

Fig 2: (top) Spherically averaged temperatures in every $10 \AA$-shell at $t=8,24$, and 40ps. (middle) Same as (top) but for densities of O. (bottom) Same as (top) but for densities of $\mathrm{Al}$.

Oxygen atoms located closer than $\sim 4 \AA$ from the surface of the Al cluster are strongly attracted by the cluster. In the early stage of oxidation ( $t=0-25 \mathrm{ps})$, we find that the thickness of the surface oxides increases linearly with time. Owing to the release of the bond-formation 
energy between $\mathrm{O}$ and $\mathrm{Al}$, the surface temperature rises linearly with time until it reaches $\sim 2,300 \mathrm{~K}$ at $t=24 \mathrm{ps}$ as seen in Fig. 2 (top). This is close to the melting temperature of alumina (i.e., 2,327K). Significant diffusions of $\mathrm{Al}$ and $\mathrm{O}$ along the tangential direction to the surface are observed in the melted surface-oxides: for $O$ atoms in the range $r=90-100 \AA$ at $t=24 \mathrm{ps}, \Delta r_{1}^{2} / \Delta t \sim$ $14 \times 10^{-4} \mathrm{~cm}^{2} / \mathrm{s}$ for tangential and $\Delta r_{\mathrm{r}}^{2} / \Delta t \sim 7 \times 10^{-4} \mathrm{~cm}^{2} / \mathrm{s}$ for radial motions. Radial diffusion toward the center is suppressed because of lower temperatures at smaller radii. Uniformity in the oxidation thickness at various polar angles observed in Figs. 1 results from such enhanced surface-diffusion.

For $t=25-35 \mathrm{ps}$, the surface oxides reach a thickness $\sim 28 \AA$. One of the reasons for the saturation of the oxide thickness is that $O$ atoms are depleted near the surface of the cluster $(r \sim 120 \AA)$ as shown in Fig. 2 (middle). It is known that suboxides such as AlO are stable in the gaseous state at temperatures higher than $\sim 1,900 \mathrm{~K}$ [16]. In the present simulation, the temperatures at $r \sim 120 \AA$ are in fact higher than $1,900 \mathrm{~K}$ for $t>24 \mathrm{ps}$ as shown in Fig. 2 (top). Therefore, a possible reason for such a depletion of $O$ near the surface is that further increase in $n_{0}$ is hindered since $n_{0} / n_{A I}-1$ near the surface of the cluster at $t \sim 24 p s$. Disordering of the crystalline structure is observed to develop from the surface, which extends to $r \sim 50 \AA$ at $t=32 \mathrm{ps}$ (see, Fig. 1). It is because crystalline Al melts at temperatures higher than $\sim 1,000 \mathrm{~K}$.

For 140 ps, we observe in Fig. 1 thermal emission of fragmented oxides of various sizes. The surface temperature is higher than $3,000 \mathrm{~K}$ for $t>40 \mathrm{ps}$ as depicted in Fig. 2 (top). The entire cluster melts at $t \sim 55 \mathrm{ps}$.

\section{VIBRATIONAL PROPERTIES OF OXIDIZED ALUMINUM CLUSTERS}

Laboratory experiments $[2,3]$ show that $\mathrm{Al}$ nanoclusters form amorphous- $\mathrm{Al}_{\mathrm{x}} \mathrm{O}_{\mathrm{y}}$ surface layers of thickness $20-30 \AA$ in the environmental oxygen gas at room temperatures. Therefore, we quench the melted surface-oxides to form solid oxides in the surface region of the cluster. Subsequently, we calculate vibrational density-of-states (DOS) in various radial shells of the cluster. These are compared with vibrational spectra for crystalline $\mathrm{Al}, \mathrm{Al}$ cluster, and $\alpha-\mathrm{Al}_{2} \mathrm{O}_{3}$.

Starting with the atomic configuration at $t=35 \mathrm{ps}$, we quench by scaling the velocities 0.99 at intervals of $20 \mathrm{fs}$. After $20 \mathrm{ps}$, we isolate the cluster region $(r<120 \AA)$ from the total system and apply the steepest descent method to obtain the final local-equilibrium configuration. We find amorphous surface-oxides of thickness $20-25 \AA$ covering the $A$ l core. Averaged densities in the midst of the oxides ( $r=90-100 \AA$ ) are $n_{\mathrm{Al}}=4.37 \times 10^{-2} \AA^{-3}$ for $\mathrm{Al}$ and $n_{0}=1.56 \times 10^{-2} \AA^{-3}$ for O. Hence the averaged mass density is $2.4 \mathrm{~g} / \mathrm{cm}^{3}$, which is smaller than $4.0 \mathrm{~g} / \mathrm{cm}^{3}$ for $\alpha-\mathrm{Al}_{2} \mathrm{O}_{3}$ and $2.7 \mathrm{~g} / \mathrm{cm}^{3}$ for crystalline Al.

Normalized vibrational-frequency DOS for the oxidized cluster is calculated by Fourier transforming the velocity auto-correlation functions at 100K. Figure 3 (left) compares the DOS's calculated in various radial shells: $r=70-80,80-90,90-100$, and $100-110 \AA$. In the shell $70-80 \AA$

where $n_{\mathrm{O}} / n_{\mathrm{Al}}=0.01$, prominent peaks are observed at $\hbar \omega \sim 17$ and $35 \mathrm{meV}$. In the shell $80-90 \AA$, a broad spectrum ranging from $\hbar \omega=50-120 \mathrm{meV}$ emerges while the widths of the two peaks ( $\hbar \omega \sim 17$ and $35 \mathrm{meV}$ ) observed in the shell $70-80 \AA$ increase. Such a broad spectrum further extends to the range $\hbar \omega=50-170 \mathrm{meV}$ in the shells $90-100$ and $100-110 \AA$.

To understand these features in the DOS's, we compare in Fig. 3 (right) the DOS for the surface oxides ( $r=80-110 \AA$ ) with those for crystalline $\mathrm{Al}$ and $\alpha-\mathrm{Al}_{2} \mathrm{O}_{3}$. For crystalline $\mathrm{Al}$, we have performed MD simulations of 864-atom fcc crystal using the embedded-atom interatomic potentials [7] and obtained the DOS from the velocity auto-correlation functions. The DOS for crystalline Al has two sharp peaks at $\hbar \omega \sim 17$ and $32 \mathrm{meV}$ as shown in Fig. 3 (right). We have also calculated the DOS for $\mathrm{Al}$ nanocluster of radius $100 \AA$ and we find that it is indiscernible from that for crystalline Al. The DOS for $\alpha-\mathrm{Al}_{2} \mathrm{O}_{3}$ in Fig. 3 (right) is taken from ref. [11]. It was 
calculated by diagonalizing the dynamical matrix in which the ES+ interatomic potentials were adopted. There is reasonable agreement between. such theoretical prediction and experimental data obtained by the neutron scattering technique [11].

We find through comparisons of the DOS's in Fig. 3 (right) that the two peaks at $\hbar \omega \sim 17$ and $35 \mathrm{meV}$ observed for the oxidized cluster correspond to those for crystalline Al. The broad spectra ( $\hbar \omega=50-120 \mathrm{meV}$ ) observed in the shell $80-90 \AA$ of the oxidized cluster resembles the DOS for $\alpha-\mathrm{Al}_{2} \mathrm{O}_{3}$. The high frequencies ( $\hbar \omega=110-180 \mathrm{meV}$ ) observed for the shells $90-100$ and 100-110 $\AA$ of the oxidized cluster have no correspondence in the DOS's for either crystalline Al or $\alpha-\mathrm{Al}_{2} \mathrm{O}_{3}$.

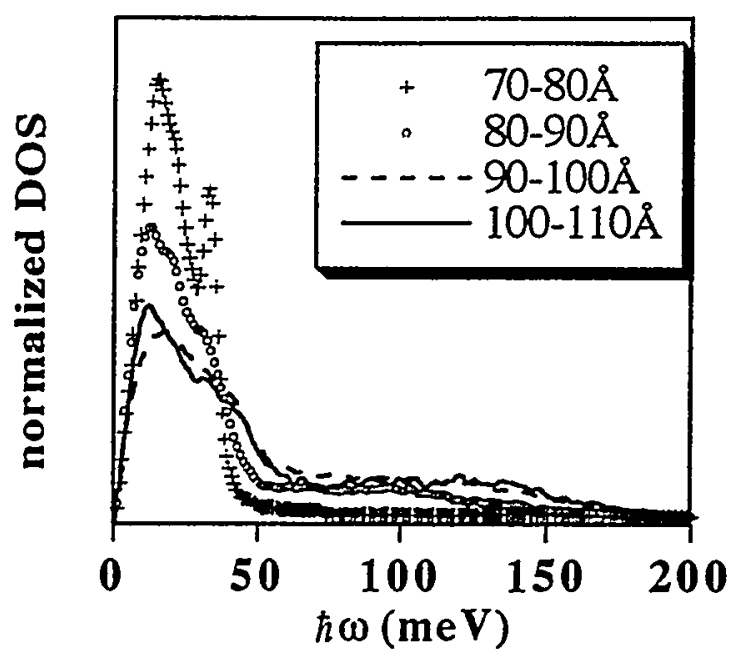

Fig 3: (left) Vibrational DOS in various radial shells for the oxidized $\mathrm{Al}$ cluster.

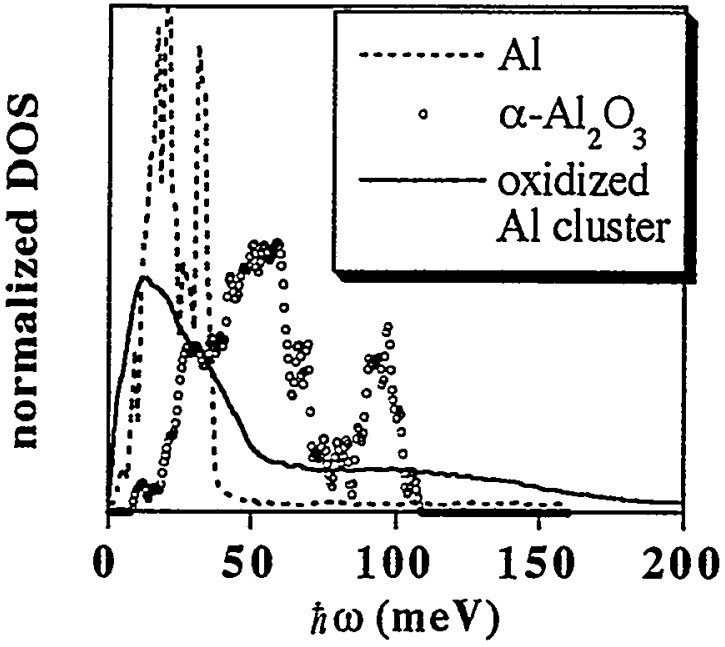

(right) Comparisons of DOS's for the oxidized $\mathrm{Al}$ cluster, crystalline $\mathrm{Al}$, and $\alpha-\mathrm{Al}_{2} \mathrm{O}_{3}$.

\section{CONCLUSIONS}

We have performed large-scale $\mathrm{MD}$ simulations for the oxidation of an $\mathrm{Al}$ nanocluster of radius $100 \AA$ on parallel computers. The simulations take into account the effect of charge transfer between $\mathrm{Al}$ and $\mathrm{O}$ based on the electronegativity equalization principles. We have found that the oxidation starts at the surface of the cluster and the oxide layer grows until it saturates to a thickness of $\sim 28 \AA$ at 35ps. Local temperature in the surface region due to the release of energy when Al-O bonds form, melts the surface oxide. Amorphous surface-oxides have been obtained by quenching the cluster. Vibrational DOS's for the amorphous oxides have characteristic features of both crystalline $\mathrm{Al}$ and $\alpha-\mathrm{Al}_{2} \mathrm{O}_{3}$.

\section{ACKNOWLEDGMENTS}

One of us (TJC) is supported by the NSF Graduate Research Traineeship (Grant No. DGE-9355007). CKL is supported by by U.S. Department of Energy, BES, Grant No. W-31109-ENG-38. This work was supported by the U.S. Department of Energy, Grant No. DEFG05-92ER45477, National Science Foundation, Grant No. DMR-9412965 and ASC-9701504, Air Force Office of Scientific Research, Grant No. F 49620-94-1-0444, Army Research Office, Grant No. DAAH04-96-1-0393, Louisiana Education Quality Support Fund (LEQSF), Grant No. LEQSF96-99-RD-A-10, USC-LSU Multidisciplinary University Research Initiative, Grant No. F 49620-95-1-0452. and Petroleum Research Fund, Grant No. 31659-AC9. The computations 
were performed on parallel machines in the Concurrent Computing Laboratory for Materials Simulations (CCLMS) at Louisiana State University. The facilities in the CCLMS were acquired with the Equipment Enhancement Grants awarded by the Louisiana Board of Regents through Louisiana Education Quality Support Fund (LEQSF).

\section{REFERENCES}

1. e.g., D. R. Askeland, The Science and Engineering of Materials, (PWS-KENT Pub. Co., Boston, 1989), Chap. 20.

2. S. Sako, K. Ohshima, and T. Fujita, J. Phys. Soc. Jpn. 59, 662 (1990).

3. C. E. Aumann, G. L. Skofronick, and J. A. Martin, J. Vac. Sco. Technol. B 13, 1178 (1995).

4. B. H. Suits, P. Apte, D. E. Wilken, and R. W. Siegel, Nanostruc. Mat. 6, 609 (1995).

5. J. C. Sánchez-López, A. Fernández, C. F. Conde, A. Conde, C. Morant, and J. M. Sanz, Nanostruct. Mat. 7, 813 (1996).

6. T. G. Nieh, P. Luo, W. Nellis, D. Lesuer, and D. Benson, Acta. Mater. 44, 3781 (1996).

7. F. H. Streitz and J. W. Mintmire, Phys. Rev. B 50, 11996 (1994).

8. S. W. Rick, S. J. Stuart, and B. J. Berne, J. Chem. Phys. 101, 614 (1994).

9. e.g., R. G. Parr and R. G. Pearson, J. Am. Chem. Soc. 105, 7512 (1983).

10. I. Manassidis, A. DeVita, and M. J. Gillan, Surf. Sci. Lett. 285, L517 (1993).

11. T. J. Campbell, A. Nakano, R. K. Kalia, P. Vashishta, and C.-K. Loong, private comminications.

12. L. Greengard and V. Rokhlin, J. Comp. Phys. 73, 325 (1987).

13. C. A. White and M. Head-Gordon, J. Chem. Phys. 101, 6593 (1994).

14. S. Ogata et al., to be published.

15. M. P. Allen and T. J. Tildesley, Computer Simulation of Liquids, (Oxford Univ. Press, 1990).

16. Z. Katz-Tsameret and A. Raveh, J. Vac. Sci. Technol. A 13, 1121 (1995). 